\title{
Some extremal problems for hereditary properties of graphs
}

\author{
Vladimir Nikiforov \\ Department of Mathematical Sciences \\ University of Memphis, Memphis, TN, USA \\ vnikifrv@memphis.edu
}

Submitted: Jun 1, 2013; Accepted: Jan 13, 2014; Published: Jan 24, 2014

Mathematics Subject Classifications: 05C65, 05C35

\begin{abstract}
Given an infinite hereditary property of graphs $\mathcal{P}$, the principal extremal parameter of $\mathcal{P}$ is the value

$$
\pi(\mathcal{P})=\lim _{n \rightarrow \infty}\left(\begin{array}{l}
n \\
2
\end{array}\right)^{-1} \max \{e(G): G \in \mathcal{P} \text { and } v(G)=n\} .
$$

The Erdös-Stone theorem gives $\pi(\mathcal{P})$ if $\mathcal{P}$ is monotone, but this result does not apply to hereditary $\mathcal{P}$. Thus, one of the results of this note is to establish $\pi(\mathcal{P})$ for any hereditary property $\mathcal{P}$.

Similar questions are studied for the parameter $\lambda^{(p)}(G)$, defined for every real number $p \geqslant 1$ and every graph $G$ of order $n$ as

$$
\lambda^{(p)}(G)=\max _{\left|x_{1}\right|^{p}+\cdots+\left|x_{n}\right|^{p}=1} 2 \sum_{\{u, v\} \in E(G)} x_{u} x_{v} .
$$

It is shown that the limit

$$
\lambda^{(p)}(\mathcal{P})=\lim _{n \rightarrow \infty} n^{2 / p-2} \max \left\{\lambda^{(p)}(G): G \in \mathcal{P} \text { and } v(G)=n\right\}
$$

exists for every hereditary property $\mathcal{P}$.

A key result of the note is the equality

$$
\lambda^{(p)}(\mathcal{P})=\pi(\mathcal{P})
$$

which holds for all $p>1$. In particular, edge extremal problems and spectral extremal problems for graphs are asymptotically equivalent.

Keywords: extremal problems; Turán problems; hereditary property; largest eigenvalue.
\end{abstract}




\section{Introduction and main results}

In this note we study problems stemming from the following one:

Problem 1. What is the maximum number of edges in a graph $G$ of order $n$ if $G$ belongs to some hereditary property $P$.

Let us recall the basics of graph properties: A graph property is just a family of graphs closed under isomorphisms. A property is called monotone if it is closed under taking subgraphs, and hereditary if it is closed under taking induced subgraphs. Given a set of graphs $\mathcal{F}$, the family of all graphs that do not contain any $F \in \mathcal{F}$ as a subgraph is a monotone property, denoted by $\operatorname{Mon}(\mathcal{F})$. Likewise, the family of all graphs that do not contain any $F \in \mathcal{F}$ as an induced subgraph is a hereditary property, denoted as $\operatorname{Her}(\mathcal{F})$.

It seems that the classically shaped Problem 1 has been disregarded in the rich literature on hereditary properties, so in this paper we shall fill in this gap. Note, however, that for monotone properties the theorem of Erdős and Stone provides a well-known solution, outlined in Proposition 3 below.

Writing $\mathcal{P}_{n}$ for the set of all graphs of order $n$ in a property $\mathcal{P}$, now Problem 1 reads as:

Given a hereditary property $\mathcal{P}$, find

$$
\operatorname{ex}(\mathcal{P}, n)=\max _{G \in \mathcal{P}_{n}} e(G) .
$$

Finding ex $(\mathcal{P}, n)$ exactly seems hopeless for arbitrary $\mathcal{P}$. A more feasible approach has been suggested by Katona, Nemetz and Simonovits in [8] who proved the following fact:

Proposition 2. If $\mathcal{P}$ is a hereditary property, then the limit

$$
\pi(\mathcal{P})=\lim _{n \rightarrow \infty} \operatorname{ex}(\mathcal{P}, n)\left(\begin{array}{l}
n \\
2
\end{array}\right)^{-1}
$$

exists.

In particular, for monotone properties Erdős and Simonovits [6] observed the following consequence of the Erdös-Stone theorem [5]:

Proposition 3. If a monotone property $\mathcal{P}$ is given as $\mathcal{P}=\operatorname{Mon}(\mathcal{F})$ for some nonempty family $\mathcal{F}$, then

$$
\pi(\operatorname{Mon}(\mathcal{F}))=1-1 / \chi
$$

where $\chi=\min \{\chi(F): F \in \mathcal{F}\}$.

Unfortunately, $\pi(\mathcal{P})$ cannot be determined in the same simple way for a general hereditary property $\mathcal{P}$, and so one of the aims of this note is to establish $\pi(\mathcal{P})$ for such $\mathcal{P}$. However, our main focus is on extremal problems about a more general graph parameter, denoted by $\lambda^{(p)}(G)$ and defined as follows: 
Given a graph $G$ and a real number $p \geqslant 1$, let

$$
\lambda^{(p)}(G)=\max _{\left|x_{1}\right|^{p}+\cdots+\left|x_{n}\right|^{p}=1} 2 \sum_{\{u, v\} \in E(G)} x_{u} x_{v} .
$$

Note that $\lambda^{(2)}(G)$ is the well-studied spectral radius of $G$, and also that $\lambda^{(1)}(G)$ is a another much studied graph parameter, known as the Lagrangian ${ }^{1}$ of $G$. Moreover, letting $p \rightarrow \infty$, one can show that $\lambda^{(p)}(G) \rightarrow e(G)$. So $\lambda^{(p)}(G)$ is a common generalization of three central parameters in extremal graph theory.

The parameter $\lambda^{(p)}(G)$ has been introduced and studied for uniform hypergraphs first by Keevash, Lenz and Mubayi in [7], and next by the author in [12] and [13]. Here we shall study $\lambda^{(p)}(G)$ in the same role as $e(G)$ in equation (1), obtaining thus the following problem.

Problem 4. Given a hereditary property $\mathcal{P}$, find

$$
\lambda^{(p)}(\mathcal{P}, n)=\max _{G \in \mathcal{P}_{n}} \lambda^{(p)}(G) .
$$

As for $\operatorname{ex}(\mathcal{P}, n)$, finding $\lambda^{(p)}(\mathcal{P}, n)$ seems hopeless for arbitrary $\mathcal{P}$, so we begin with an analog to Proposition 2.

Theorem 5. Let $p \geqslant 1$. If $\mathcal{P}$ is a hereditary property, then the limit

$$
\lambda^{(p)}(\mathcal{P})=\lim _{n \rightarrow \infty} \lambda^{(p)}(\mathcal{P}, n) n^{(2 / p)-2}
$$

exists.

The main goal of this note is to find $\lambda^{(p)}(\mathcal{P})$ for every $\mathcal{P}$ and every $p \geqslant 1$. It turns out that $\lambda^{(p)}(\mathcal{P})$ and $\pi(\mathcal{P})$ are almost identical. Indeed, general results proved in [12] imply that $\lambda^{(p)}(\mathcal{P})=\pi(\mathcal{P})$ for every $p>1$, and also $\lambda^{(1)}(\mathcal{P}) \geqslant \pi(\mathcal{P})$. In this note we give an alternative direct proof of these results, and we find $\pi(\mathcal{P})$ explicitly.

Before going further we need some definitions. Recall that a complete $r$-partite graph is a graph whose vertices can be split into $r$ nonempty independent sets so that all edges between vertices of different classes are present. In particular, an 1-partite graph is just a set of isolated vertices.

Further, note that every hereditary property $\mathcal{P}$ can be represented as $\mathcal{P}=\operatorname{Her}(\mathcal{F})$ for some family $\mathcal{F}$, so hereafter we shall assume that every hereditary property is given as $\mathcal{P}=\operatorname{Her}(\mathcal{F})$ for some explicit family $\mathcal{F}$.

Next, for every family of graphs $\mathcal{F}$, define the parameters $\underline{\omega}(\mathcal{F})$ and $\beta(\mathcal{F})$ as

$$
\begin{aligned}
& \underline{\omega}(\mathcal{F})=\left\{\begin{array}{l}
0, \text { if } \mathcal{F} \text { contains no cliques; } \\
\min \left\{r: K_{r} \in \mathcal{F}\right\}, \text { otherwise. }
\end{array}\right. \\
& \beta(\mathcal{F})=\left\{\begin{array}{l}
0, \text { if } \mathcal{F} \text { contains no complete partite graphs; } \\
\min \{r: \mathcal{F} \text { contains a complete } r \text {-partite graph }\}, \text { otherwise. }
\end{array}\right.
\end{aligned}
$$

\footnotetext{
${ }^{1}$ Let us note that this use of the name Lagrangian is at odds with the tradition. Indeed, names as Laplacian, Hessian, Gramian, Grassmanian, etc., usually denote a structured object like matrix, operator, or manifold, and not just a single number.
} 
The parameters $\underline{\omega}(\mathcal{F})$ and $\beta(\mathcal{F})$ are quite informative about the hereditary property $\operatorname{Her}(\mathcal{F})$ as seen in the following observation.

Proposition 6. If the property $\mathcal{P}=\operatorname{Her}(\mathcal{F})$ is infinite, then $\underline{\omega}(\mathcal{F})=0$ or $\underline{\omega}(\mathcal{F}) \geqslant 2$ and $\beta(\mathcal{F}) \geqslant 2$.

Proof. Suppose that $\underline{\omega}(\mathcal{F}) \neq 0$. If $\underline{\omega}(\mathcal{F})=1$, then $\mathcal{P}$ is empty, so we shall suppose that $\underline{\omega}(\mathcal{F}) \geqslant 2$. This implies that $\beta(\mathcal{F})>0$, as $\mathcal{F}$ contains $K_{r}$ for some $r \geqslant 2$ and $K_{r}$ is a complete $r$-partite graph. If $\beta(\mathcal{F})=1$, then $\mathcal{F}$ contains a graph $F$ consisting of isolated vertices, let say $s$ be the order of $F$. Choose a member $G \in \mathcal{P}$ with $v(G) \geqslant r\left(K_{r}, K_{s}\right)$, where $r\left(K_{r}, K_{s}\right)$ is the Ramsey number of $K_{r}$ vs. $K_{s}$. Thus, $G$ contains either a $K_{r}$ or an independent set on $s$ vertices, both of which are forbidden. This contradiction shows that $\beta(\mathcal{F}) \geqslant 2$, proving Proposition 6 .

Clearly the study of (1) and (2) makes sense only if $\mathcal{P}$ is infinite, and Proposition 6 provides a necessary condition for this feature of $\mathcal{P}$. Now, the following theorem completely determines $\pi(\mathcal{P})$.

Theorem 7. Let $\mathcal{F}$ be a family of graphs. If the property $\mathcal{P}=\operatorname{Her}(\mathcal{F})$ is infinite, then

$$
\pi(\mathcal{P})=\left\{\begin{array}{ll}
1, & \text { if } \underline{\omega}(\mathcal{F})=0 \\
1-\frac{1}{\beta(\mathcal{F})-1}, & \text { otherwise. }
\end{array} .\right.
$$

Let us turn now to the study of $\lambda^{(p)}(\mathcal{P})$. As mentioned above, in [12] it has been proved that $\pi(\mathcal{P})=\lambda^{(p)}(\mathcal{P})$ for $p>1$; however, for reader's sake we shall establish this identity directly.

Theorem 8. Let $p>1$ and let $\mathcal{F}$ be a family of graphs. If the property $\mathcal{P}=\operatorname{Her}(\mathcal{F})$ is infinite, then

$$
\lambda^{(p)}(\mathcal{P})=\left\{\begin{array}{ll}
1, & \text { if } \underline{\omega}(\mathcal{F})=0 ; \\
1-\frac{1}{\beta(\mathcal{F})-1}, & \text { otherwise. }
\end{array} .\right.
$$

To complete the description of $\lambda^{(p)}(\mathcal{P})$ we need to determine the dependence of $\lambda^{(1)}(\mathcal{P})$ on $\mathcal{P}$. Using the well-known idea of Motzkin and Straus [9], we come up with the following theorem, whose easy proof we omit.

Theorem 9. If $\mathcal{P}$ is an infinite hereditary property, then $\lambda^{(1)}(\mathcal{P})=1-1 / r$ if $r$ is the size of the largest clique in $\mathcal{P}$, and $\lambda^{(1)}(\mathcal{P})=1$ if $\mathcal{P}$ contains arbitrary large cliques.

The next section contains proofs of Theorems 5,7 and 8 , and some auxiliary statements. In the final section we outline a line of possible future research. 


\section{Proofs}

\subsection{Some preliminary results}

Below we state several results necessary for the proof of our key Theorem 8 . The first one follows from a result in [12], but for reader's sake we give its short proof here.

Theorem 10. Let $p \geqslant 1$. If $G$ is a graph with $m$ edges and $n$ vertices, with no $K_{r+1}$, then

$$
\lambda^{(p)}(G) \leqslant\left(1-\frac{1}{r}\right)^{1 / p}(2 m)^{1-1 / p}
$$

and

$$
\lambda^{(p)}(G) \leqslant\left(1-\frac{1}{r}\right) n^{2-2 / p}
$$

Proof. Indeed, let $\mathbf{x}=\left(x_{1}, \ldots, x_{n}\right)$ be a vector such that $\left|x_{1}\right|^{p}+\cdots+\left|x_{n}\right|^{p}=1$ and

$$
\lambda^{(p)}(G)=2 \sum_{\{u, v\} \in E(G)} x_{u} x_{v} .
$$

Applying the Power Mean Inequality, we see that

$$
\begin{aligned}
\lambda^{(p)}(G) & =2 \sum_{\{u, v\} \in E(G)} x_{u} x_{v} \leqslant 2 \sum_{\{u, v\} \in E(G)}\left|x_{u}\right|\left|x_{v}\right| \\
& \leqslant(2 m)^{1-1 / p}\left(2 \sum_{\{u, v\} \in E(G)}\left|x_{u}\right|^{p}\left|x_{v}\right|^{p}\right)^{1 / p} .
\end{aligned}
$$

Now, the result of Motzkin and Straus [9] impies that

$$
2 \sum_{\{u, v\} \in E(G)}\left|x_{u}\right|^{p}\left|x_{v}\right|^{p} \leqslant 1-\frac{1}{r},
$$

and inequality (3) follows. Finally, inequality (4) follows from (3) by Turán's inequality $2 m \leqslant(1-1 / r) n^{2}$.

Note, in particular, that $\lambda^{(p)}(G) \leqslant(2 m)^{1-1 / p}$. This simple bound will be used in the proof of the following proposition.

Proposition 11. Let $p \geqslant 1, k>1$ and $G_{1}$ and $G_{2}$ be graphs on the same vertex set. If $G_{1}$ and $G_{2}$ differ in at most $k$ edges, then

$$
\left|\lambda^{(p)}\left(G_{1}\right)-\lambda^{(p)}\left(G_{2}\right)\right| \leqslant(2 k)^{1-1 / p} .
$$


Proof. Let $V=V\left(G_{1}\right)=V\left(G_{2}\right)$ and write $G_{12}$ for the graph with $V\left(G_{12}\right)=V$ and $E\left(G_{12}\right)=E\left(G_{1}\right) \cap E\left(G_{2}\right)$. We may and shall assume that $\lambda^{(p)}\left(G_{1}\right) \geqslant \lambda^{(p)}\left(G_{2}\right)$. Write $G_{3}$ for the graph with $V\left(G_{3}\right)=V$ and $E\left(G_{3}\right)=E\left(G_{1}\right) \backslash E\left(G_{2}\right)$. In view of $G_{12} \subset G_{2}$, we have

$$
\begin{aligned}
0 & \leqslant \lambda^{(p)}\left(G_{1}\right)-\lambda^{(p)}\left(G_{2}\right)=\lambda^{(p)}\left(G_{1}\right)-\lambda^{(p)}\left(G_{12}\right)-\left(\lambda^{(p)}\left(G_{2}\right)-\lambda^{(p)}\left(G_{12}\right)\right) \\
& \leqslant \lambda^{(p)}\left(G_{1}\right)-\lambda^{(p)}\left(G_{12}\right) \leqslant \lambda^{(p)}\left(G_{3}\right) \leqslant\left(2 e\left(G_{3}\right)\right)^{1-1 / p} \\
& \leqslant(2 k)^{1-1 / p}
\end{aligned}
$$

proving Proposition 11.

Further, let us recall the following particular version of the Removal Lemma, which is a consequence of the Szemerédi Regularity Lemma ([15], [1]):

Removal Lemma For all $r \geqslant 3$ and $\varepsilon>0$, there exists $\delta=\delta(r, \varepsilon)>0$ such that if $G$ is a graph of order $n$, with $k_{r}(G)<\delta n^{r}$, then there is a graph $G_{0} \subset G$ such that $e\left(G_{0}\right) \geqslant e(G)-\varepsilon n^{2}$ and $k_{r}\left(G_{0}\right)=0$.

Finally, we shall need the following theorem proved in [10]:

Theorem A For all $r \geqslant 2$ and $\varepsilon>0$, there exists $\delta=\delta(r, \varepsilon)>0$ such that if $G$ a graph of order $n$ with $k_{r}(G)>\varepsilon n^{r}$, then $G$ contains a $K_{r}(s)$ with $s=\lfloor\delta \log n\rfloor$.

\subsection{Proof of Theorem 5}

Proof. Set for short $\lambda_{n}^{(p)}=\lambda^{(p)}(\mathcal{P}, n)$. Let $G \in \mathcal{P}_{n}$ be such that $\lambda_{n}^{(p)}=\lambda^{(p)}(G)$ and let $\mathbf{x}=\left(x_{1}, \ldots, x_{n}\right)$ be a vector with $\left|x_{1}\right|^{p}+\cdots+\left|x_{n}\right|^{p}=1$ and

$$
\lambda_{n}^{(p)}=\lambda^{(p)}(G)=2 \sum_{\{u, v\} \in E(G)} x_{u} x_{v}
$$

If $p=1$, we obviously have $\lambda_{n}^{(1)} \geqslant \lambda_{n-1}^{(1)}$ and in view of

$$
\lambda_{n}^{(1)}=2 \sum_{\{u, v\} \in E(G)} x_{u} x_{v} \leqslant 2 \sum_{1 \leqslant i<j \leqslant n} x_{i} x_{j}<\left(x_{1}+\cdots+x_{n}\right)^{2}=1,
$$

the sequence $\left\{\lambda_{n}^{(1)}\right\}_{n=1}^{\infty}$ converges to some $\lambda$. We have

$$
\lambda=\lim _{n \rightarrow \infty} \lambda_{n}^{(1)} n^{2-2}=\lambda^{(1)}(\mathcal{P}),
$$

proving the theorem for $p=1$.

Now suppose that $p>1$. Since $\left|x_{1}\right|^{p}+\cdots+\left|x_{n}\right|^{p}=1$, there is a vertex $k \in V(G)$ such that $\left|x_{k}\right|^{p} \leqslant 1 / n$. Write $G-k$ for the graph obtained from $G$ by omitting the vertex $k$, 
and let $\mathbf{x}^{\prime}=\left(x_{1}^{\prime}, \ldots, x_{n-1}^{\prime}\right)$ be the $(n-1)$-vector obtained from $\mathbf{x}$ by omitting the entry $x_{k}$. The eigenequation for $\lambda^{(p)}(G)$ and the vertex $k$ is

$$
\lambda^{(p)}(G) x_{k}\left|x_{k}\right|^{p-2}=\sum_{\{k, i\} \in E(G)} x_{i} .
$$

Hence, we see that

$$
\begin{aligned}
2 \sum_{\{u, v\} \in E(G-k)} x_{u}^{\prime} x_{v}^{\prime} & =2 \sum_{\{u, v\} \in E(G)} x_{u} x_{v}-2 x_{k} \sum_{\{k, i\} \in E(G)} x_{i} \\
& =\lambda^{(p)}(G)-2 x_{k}\left(\lambda^{(p)}(G) x_{k}\left|x_{k}\right|^{p-2}\right)=\lambda_{n}^{(p)}\left(1-2\left|x_{k}\right|^{p}\right) .
\end{aligned}
$$

Since $\mathcal{P}$ is a hereditary property, $G-k \in \mathcal{P}_{n-1}$, and therefore,

$$
2 \sum_{\{u, v\} \in E(G-k)} x_{u}^{\prime} x_{v}^{\prime} \leqslant \lambda^{(p)}(G-k)\left|\mathbf{x}^{\prime}\right|_{p}^{2}=\lambda^{(p)}(G-k)\left(1-\left|x_{k}\right|^{p}\right)^{2 / p} \leqslant \lambda_{n-1}^{(p)}\left(1-\left|x_{k}\right|^{p}\right)^{2 / p} .
$$

Thus, we obtain

$$
\lambda_{n}^{(p)} \leqslant \lambda_{n-1}^{(p)} \frac{\left(1-\left|x_{k}\right|^{p}\right)^{2 / p}}{\left(1-2\left|x_{k}\right|^{p}\right)} .
$$

Note that the function

$$
f(y)=\frac{(1-y)^{2 / p}}{1-2 y}
$$

is nondecreasing in $y$ for $0 \leqslant y \leqslant 1 / n$ and $n$ sufficiently large. Indeed,

$$
\begin{aligned}
\frac{d f(y)}{d y} & =\frac{-\frac{2}{p}(1-y)^{2 / p-1}(1-2 y)+2(1-y)^{2 / p}}{(1-2 y)^{2}} \\
& =\left(-\frac{1}{p}(1-2 y)+(1-y)\right) \frac{2(1-y)^{2 / p-1}}{(1-2 y)^{2}} \\
& =\left(-\left(\frac{1}{p}-1\right)+\left(\frac{2}{p}-1\right) y\right) \frac{2(1-y)^{2 / p-1}}{(1-2 y)^{2}} \geqslant 0
\end{aligned}
$$

Here we use the fact that $1 / p-1>0$ and that $(2 / p-1) y$ tends to 0 when $n \rightarrow \infty$.

Hence, in view of (5), we find that for $n$ large enough

$$
\lambda_{n}^{(p)} \leqslant \lambda_{n-1}^{(p)} f\left(\left|x_{k}\right|^{p}\right) \leqslant \lambda_{n-1}^{(p)} f\left(\frac{1}{n}\right)=\lambda_{n-1}^{(p)} \frac{n(1-1 / n)^{2 / p}}{(n-2)},
$$

and so

$$
\frac{\lambda_{n}^{(p)} n^{2 / p}}{n(n-1)} \leqslant \frac{\lambda_{n-1}^{(p)}(n-1)^{2 / p}}{(n-1)(n-2)}
$$

Therefore, the sequence

$$
\left\{\frac{\lambda_{n}^{(p)} n^{2 / p}}{n(n-1)}\right\}_{n=1}^{\infty}
$$

is nonincreasing, and so it is converging, completing the proof of Theorem 5 . 


\subsection{Proof of Theorem 7}

Proof. Since $\mathcal{P}$ is infinite, Proposition 6 implies that $\underline{\omega}(\mathcal{F})=0$ or $\underline{\omega}(\mathcal{F}) \geqslant 2$ and $\beta(\mathcal{F}) \geqslant$ 2. If $\underline{\omega}(\mathcal{F})=0$, then $K_{n} \in \mathcal{P}_{n}$, because all induced subgraphs of $K_{n}$ are complete and therefore do not belong to $\mathcal{F}$. Hence,

$$
\operatorname{ex}(\mathcal{P}, n)=\left(\begin{array}{l}
n \\
2
\end{array}\right)
$$

and so, $\pi(\mathcal{P})=1$.

Now assume that $r=\underline{\omega}(\mathcal{F}) \geqslant 2$ and $\beta=\beta(\mathcal{F}) \geqslant 2$. We shall prove that $T_{\beta-1}(n) \in \mathcal{P}_{n}$, where $T_{\beta-1}(n)$ is the complete $(\beta-1)$-partite Turán graph of order $n$. Indeed all induced subgraphs of $T_{\beta-1}(n)$ are complete $r$-partite graphs for some $r \leqslant \beta-1$, so should one of them belong to $\mathcal{F}$, we would have $\beta(\mathcal{F}) \leqslant \beta-1=\beta(\mathcal{F})-1$, which is a contradiction. Therefore,

$$
\operatorname{ex}(\mathcal{P}, n) \geqslant e\left(T_{\beta-1}(n)\right)=\left(1-\frac{1}{\beta-1}+o(1)\right)\left(\begin{array}{l}
n \\
2
\end{array}\right),
$$

and so

$$
\pi(\mathcal{P}) \geqslant 1-\frac{1}{\beta(\mathcal{F})-1} .
$$

To finish the proof we shall prove the opposite inequality. Let $F \in \mathcal{F}$ be a complete $\beta$-partite graph, which exists by the definition of $\beta(\mathcal{F})$; let $s$ be the maximum of the sizes of its vertex classes. Let $\varepsilon>0$ and set $t=r\left(K_{r}, K_{s}\right)$, where $r\left(K_{r}, K_{s}\right)$ is the Ramsey number of $K_{r}$ vs. $K_{s}$. If $n$ is large enough and $G \in \mathcal{P}_{n}$ satisfies

$$
e(G)>\left(1-\frac{1}{\beta(\mathcal{F})-1}+\varepsilon\right)\left(\begin{array}{l}
n \\
2
\end{array}\right),
$$

then, by the theorem of Erdös and Stone [5], $G$ contains a subgraph $G_{0}=K_{\beta}(t)$, that is to say, a complete $\beta$-partite graph with $t$ vertices in each vertex class. Since $K_{r} \in \mathcal{F}$, we see that $G_{0}$ contains no $K_{r}$. Hence each vertex class of $G_{0}$ contains an independent set of size $s$, and so $G$ contains an induced subgraph $K_{\beta}(s)$, which in turn contains an induced copy of $F$. Thus, if $n$ is large enough and $G \in \mathcal{P}_{n}$, then

$$
e(G)\left(\begin{array}{l}
n \\
2
\end{array}\right)^{-1} \leqslant 1-\frac{1}{\beta(\mathcal{F})-1}+\varepsilon
$$

This inequality implies that

$$
\pi(\mathcal{P}) \leqslant 1-\frac{1}{\beta(\mathcal{F})-1},
$$

completing the proof of Theorem 7 . 


\subsection{Proof of Theorem 8}

Proof. First note the inequality

$$
\lambda^{(p)}(G) \geqslant 2 e(G) / n^{2 / p}
$$

which follows by taking $\left(x_{1}, \ldots, x_{n}\right)=\left(n^{-1 / p}, \ldots, n^{-1 / p}\right)$ in $(2)$. So we see that

$$
\lambda^{(p)}(\mathcal{P}) \geqslant \pi(\mathcal{P})
$$

and this inequality together with Theorem 7 gives $\lambda^{(p)}(\mathcal{P})=1$ if $\underline{\omega}(\mathcal{F})=0$, and

$$
\lambda^{(p)}(\mathcal{P}) \geqslant 1-\frac{1}{\beta(\mathcal{F})-1}
$$

otherwise. To finish the proof we shall show that

$$
\lambda^{(p)}(\mathcal{P}) \leqslant 1-\frac{1}{\beta(\mathcal{F})-1} .
$$

For this purpose write $k_{r}(G)$ for the number of $r$-cliques of $G$. Let $F \in \mathcal{F}$ be a complete $\beta$-partite graph, which exists by the definition of $\beta(\mathcal{F})$; let $s$ be the maximum of the sizes of its vertex classes.

Now if $\varepsilon>0$ and $\varepsilon$ is sufficiently small, choose $\delta=\delta(\beta, \varepsilon)$ as in the Removal Lemma, and set $t=r\left(K_{r}, K_{s}\right)$, where $r\left(K_{r}, K_{s}\right)$ is the Ramsey number of $K_{r}$ vs. $K_{s}$. If $G \in \mathcal{P}_{n}$, then $K_{\beta}(t) \nsubseteq G$ for otherwise, as in proof of Theorem 7 , we see that $G$ contains an induced copy of $F$. So if $n$ is large enough Theorem A implies that $k_{\beta}(G) \leqslant \delta n^{r}$. Now, by the Removal Lemma, there is a graph $G_{0} \subset G$ such that $e\left(G_{0}\right) \geqslant e(G)-\varepsilon n^{2}$ and $k_{\beta}\left(G_{0}\right)=0$.

For $n$ sufficiently large Propositions 10 and 11 imply that

$$
\lambda^{(p)}(G) \leqslant \lambda^{(p)}\left(G_{0}\right)+(2 \varepsilon n)^{2-2 / p} \leqslant\left(1-\frac{1}{\beta-1}\right) n^{2-2 / p}+(2 \varepsilon n)^{2-2 / p},
$$

and hence,

$$
\lambda^{(p)}(\mathcal{P}, n) n^{2 / p-2} \leqslant 1-\frac{1}{\beta-1}+(2 \varepsilon)^{2-2 / p} .
$$

Since $\varepsilon$ can be chosen arbitrarily small, we see that

$$
\lambda^{(p)}(\mathcal{P}) \leqslant 1-\frac{1}{\beta-1},
$$

completing the proof of Theorem 8 . 


\section{Concluding remarks}

In a sequence of papers the author has shown that many classical extremal results like the Erdős-Stone-Bollobás theorem [2], the Stability Theorem of Erdős [3, 4] and Simonovits [14], and various saturation problems can be strengthened by recasting them for the largest eigenvalue instead of the number of edges; see [11] for an overview and references.

The paper [7] and the present note show that some of these edge extremal results can be extended further to $\lambda^{(p)}(G)$ for any $p>1$. A natural challenge here is to reprove all of the above problems by substituting $\lambda^{(p)}(G)$ for the number of edges.

Theorems 5, 10, and Proposition 11 have been proved in [12] for uniform hypergraphs. The streamlined proofs given here are for reader's convenience.

\section{Acknowledgement}

Thanks are due to Bela Bollobás and to Alex Sidorenko for useful discussions.

\section{References}

[1] B. Bollobás. Modern Graph Theory. Graduate Texts in Mathematics, 184, SpringerVerlag, New York (1998), xiv+394 pp.

[2] B. Bollobás and P. Erdős. On the structure of edge graphs. J. London Math. Soc., 5:317-321, 1973.

[3] P. Erdős. Some recent results on extremal problems in graph theory (Results). In Theory of Graphs (Internat. Sympos., Rome, 1966), pages 117-130. Gordon and Breach, New York; Dunod, Paris, 1967.

[4] P. Erdős. On some new inequalities concerning extremal properties of graphs. In Theory of Graphs (Proc. Colloq., Tihany, 1966), pages 77-81. Academic Press, New York, 1968.

[5] P. Erdős, A.H. Stone. On the structure of linear graphs. Bull. Amer. Math. Soc., 52:1087-1091, 1946.

[6] P. Erdős and M. Simonovits. A limit theorem in graph theory. Studia Sci. Math. Hung. 1:51-57, 1966.

[7] P. Keevash, J. Lenz, and D. Mubayi. Spectral extremal problems for hypergraphs. arXiv:1304.0050.

[8] G. Katona, T. Nemetz, and M. Simonovits. On a problem of Turán in the theory of graphs. Mat. Lapok 15:228-238, 1964.

[9] T. Motzkin and E. Straus. Maxima for graphs and a new proof of a theorem of Turán. Canad. J. Math., 17:533-540, 1965. 
[10] V. Nikiforov. Graphs with many $r$-cliques have large complete $r$-partite subgraphs. Bull. London Math. Soc., 40:23-25, 2008.

[11] V. Nikiforov. Some new results in extremal graph theory. In Surveys in Combinatorics, pages 141-181, Cambridge University Press, 2011.

[12] V. Nikiforov. An analytic theory of extremal hypergraph problems. arXiv:1305.1073v2.

[13] V. Nikiforov. Analytic methods for uniform hypergraphs. arXiv:1308.1654v3.

[14] M. Simonovits. A method for solving extremal problems in graph theory, stability problems. In Theory of Graphs (Proc. Colloq., Tihany, 1966), pages 279-319, Academic Press, New York, 1968.

[15] E. Szemerédi. Regular partitions of graphs. In Colloques Internationaux C.N.R.S. No 260 - Problèmes Combinatoires et Théorie des Graphes, pages 399-401, Orsay, 1976.

[16] P. Turán. On an extremal problem in graph theory (in Hungarian). Mat. és Fiz. Lapok, 48:436-452, 1941. 